\title{
Do Hearing Aids Improve Affect Perception?
}

\author{
Juliane Schmidt, Diana Herzog, Odette Scharenborg and Esther Janse
}

\begin{abstract}
Normal-hearing listeners use acoustic cues in speech to interpret a speaker's emotional state. This study investigates the effect of hearing aids on the perception of the emotion dimensions arousal (aroused/calm) and valence (positive/ negative attitude) in older adults with hearing loss. More specifically, we investigate whether wearing a hearing aid improves the correlation between affect ratings and affect-related acoustic parameters. To that end, affect ratings by 23 hearing-aid users were compared for aided and unaided listening. Moreover, these ratings were compared to the ratings by an age-matched group of 22 participants with age-normal hearing.

For arousal, hearing-aid users rated utterances as generally more aroused in the aided than in the unaided condition. Intensity differences were the strongest indictor of degree of arousal. Among the hearing-aid users, those with poorer hearing used additional prosodic cues (i.e., tempo and pitch) for their arousal ratings, compared to those with relatively good hearing. For valence, pitch was the only acoustic cue that was associated with valence. Neither listening condition nor hearing loss severity (differences among the hearing-aid users) influenced affect ratings or the use
\end{abstract}

\footnotetext{
J. Schmidt $(\square) \cdot$ O. Scharenborg

Centre for Language Studies, Radboud University, PO Box 9103, 6500 HD

Nijmegen, The Netherlands

e-mail: j.schmidt@let.ru.nl

O. Scharenborg

Donders Institute for Brain, Cognition and Behavior, P.O. Box 9010 // 066, 6500 GL Nijmegen, The Netherlands

e-mail: o.scharenborg@let.ru.nl

D. Herzog

Phonak AG, Laubisrütistrasse 28, 8712 Staefa, Switzerland

e-mail: Diana.Herzog@sonova.com

E. Janse

Centre for Language Studies, Radboud University, PO Box 9103, 6500 HD, Nijmegen,

The Netherlands

Donders Institute for Brain, Cognition and Behavior, P.O. Box 9010, 0666500 GL Nijmegen, The Netherlands

Max Planck Institute for Psycholinguistics, PO Box 310, 6500 AH Nijmegen, The Netherlands

(C) The Author(s) 2016

P. van Dijk et al. (eds.), Physiology, Psychoacoustics and Cognition in Normal and Impaired Hearing, Advances in Experimental Medicine and Biology 894, DOI 10.1007/978-3-319-25474-6 6
} 
of affect-related acoustic parameters. Compared to the normal-hearing reference group, ratings of hearing-aid users in the aided condition did not generally differ in both emotion dimensions. However, hearing-aid users were more sensitive to intensity differences in their arousal ratings than the normal-hearing participants.

We conclude that the use of hearing aids is important for the rehabilitation of affect perception and particularly influences the interpretation of arousal.

Keywords Emotion perception - Arousal $\cdot$ Valence $\cdot$ Affective prosody $\cdot$ Acoustic parameters $\cdot$ Natural speech $\cdot$ Older adults $\cdot$ Hearing loss $\cdot$ Hearing aids $\cdot$ Mean F0 - Mean intensity

\section{Introduction}

People use several information sources to perceive and interpret emotions. Visual information, such as facial expressions, is most informative, but auditory, prosodic cues in the speech signal also provide important cues for emotion perception. For instance, prosodic cues may alter the meaning of a spoken message, as in the case of irony: the meaning of an utterance like "I like roses" can be interpreted as positive (I do like roses) or negative (I do not like roses), depending on the applied prosody. Prosodic cues, then, are acoustic parameters in speech, such as pitch, intensity, and tempo, from which a normal-hearing listener may perceive emotion in the speech signal (Banse and Scherer 1996; Scherer 2003; Coutinho and Dibben 2013). In an ideal communicative setting both visual and auditory information is available. Everyday communication settings, however, may frequently deprive the listener of visual information, (e.g., during a telephone conversation) so that listeners have to rely on auditory information only.

As hearing loss impairs the perception of auditory information perception of prosodic information may also suffer. Although hearing aids clearly improve speech intelligibility, it is unclear to what extent hearing aids sufficiently restore information needed for emotion perception in speech. Several studies with severely hearing-impaired children and adolescents indicate that aided hearing-impaired listeners perform poorly compared to their normal-hearing peers when rating affective prosody in speech (Most et al. 1993; Most and Michaelis 2012). Moreover, they found that affect perception in hearing-impaired participants was independent of their individual hearing loss. These findings, however, cannot be directly transferred to older hearing aid wearing adults, as younger and older adults differ in the perception of affective prosody, even if both groups have normal hearing (e.g., Paulmann et al. 2008). Moreover, older adults were normal-hearing when they acquired language, and will have learned to interpret the acoustic cues associated with affect, in contrast to hearing-impaired children, who never have had a normal development of hearing and perception. Finally, the two age groups may differ in the type of hearing loss, which also complicates the comparison.

To our knowledge, in older adults only the effect of mild hearing loss has been investigated so far. Findings concerning the link between individual hearing loss 
and affect perception have been inconsistent. Orbelo and colleagues (Orbelo et al. 2005) found no effect of hearing sensitivity on affect perception, while Rigo and Lieberman (Rigo and Lieberman 1989) found that low-frequency hearing loss ( $\mathrm{PTA}_{\text {low }}$ $(0.25,0.5,1 \mathrm{kHz})>25 \mathrm{~dB} \mathrm{HL})$ impacted affect perception. Note that both these studies used acted speech. The lack of a global effect of hearing sensitivity on affect perception in these experiments could be due to the more prototypical prosodic expression of affect in acted compared to natural speech (Scherer 1986; Wilting et al. 2006). More extreme expressions of affect may be relatively easy to perceive, even for people with hearing loss (Grant 1987) thus obscuring a possible influence of hearing sensitivity on affect perception in natural communicative settings.

The current study investigates whether hearing aids restore affect perception, and how hearing loss in older adults influences affect perception. In particular, this study focuses on the question to what extent hearing aid use and hearing loss influence listeners' sensitivity to the acoustic parameters cueing affect. To that end, older (bilateral) hearing aid users are tested while wearing their hearing aid (aided condition) and without it (unaided condition). The relation between the acoustic parameters and the affect ratings are then evaluated for the two listening conditions. Moreover, the performance in the aided condition is compared to a control group of age-matched normal-hearing listeners. Participants will be tested on natural conversational speech stimuli in order to mimic realistic listening conditions.

\section{Experimental Set-up}

\subsection{Participants}

Two groups of older adults aged between 65 and 82 were tested. All participants were Swiss German native speakers and were financially compensated for their participation. The group of 23 older hearing aid users with bilaterally symmetric sensorineural hearing loss $\left(\mathrm{M}_{\text {Age }}=73.5\right.$ years, $\mathrm{SD}_{\text {Age }}=4.5 ; 17$ men, 6 women $)$ was recruited via the Phonak AG participant database. Participants have worn hearing aids bilaterally for at least 2 years. The group of 22 normal-hearing adults $\left(\mathrm{M}_{\text {Age }}\right.$ $=70.8$ years, $\mathrm{SD}_{\text {Age }}=5.2 ; 10$ men, 12 women) was recruited via the Phonak human resource department and a local senior club in Staefa, Switzerland.

Participants' hearing ability was tested by means of pure-tone audiometry (air conduction thresholds). The mean unaided pure-tone average (PTA) across 0.5, 1, 2 , and $4 \mathrm{kHz}$ for the hearing-impaired group was $49.8 \mathrm{~dB} \mathrm{HL}(\mathrm{SD}=8.7$, range: 32.5-68.8). The normal-hearing participants had age-normal thresholds (as defined in the ISO 7029:2000 standards for this age group). Thresholds below the ISO's maximum pure-tone average threshold (across $0.5,1,2$, and $4 \mathrm{kHz}$ ) at the age of 70 for men $(\mathrm{PTA}=33.5 \mathrm{~dB} \mathrm{HL})$ and women $(\mathrm{PTA}=26.0 \mathrm{~dB} \mathrm{HL})$ were considered as normal hearing. Additionally, participants underwent a brief cognitive screening test to scan for mild cognitive impairment. We used the German version of the Montreal Cognitive Assessment Test (MOCA, Nasreddine et al. 2005) using a cutoff criterion of $67 \%$ accuracy (cf. Waldron-Perrine and Axelrod 2012). The 
test was adjusted for hearing-impaired participants (Dupuis et al. 2015) by leaving out tasks in which auditorily presented items had to be memorized. All participants passed the test.

\subsection{Task and Procedure}

Affect perception was tested using the dimensional approach, in which participants indicate the level of the emotion dimensions arousal (calm vs. aroused) and valence (positive vs. negative attitude), separately on a rating scale (rather than labeling emotion categories such as "angry" or "sad").

Stimuli were short audio-only utterances from an authentic and affectively-colored German conversational speech corpus (Grimm et al. 2008). Emotion inferences from speech correlate across languages, particularly for similar languages (cf. Scherer et al. 2001). Given the close relationship between German and Swiss German, the way affect is encoded in Swiss German is not expected to differ considerably from that in German as spoken in Germany. The corpus comes with mean reference values for the degree of arousal and valence for each utterance. These reference values had been collected with a 5-step pictorial rating tool (Bradley and Lang 1994), ranging from -1 (calm/negative) to +1 (aroused/positive). The same rating tool was used to collect affective ratings in the current study. From the corpus, 24 utterances were selected for the arousal task (reference value range: -0.66 to 0.94 ) and 18 were selected for the valence task (reference value range: -0.80 to 0.77). All stimuli in our experiment were neutral regarding the content of what was said (e.g. 'Was hast du getan?' 'What have you done?') to minimize semantic interference, were shorter than $3 \mathrm{~s}$ and were produced by multiple speakers. From these two stimuli sets two randomized lists were created differing in the order in which the stimuli were presented for each emotion dimension.

Participants were comfortably seated in a sound-treated room and were tested in the free field. The pictorial rating tool was displayed on a computer screen and stimuli were presented via a single loudspeaker which was placed at head level in front of the participant $\left(0^{\circ}\right.$ azimuth $)$ at a distance of $1 \mathrm{~m}$. Participants received written and oral instructions and performed four practice trials before proceeding to the test stimuli of either rating task. Both rating tasks were completed at the participant's own pace. Utterances were rated one at a time and could be replayed if needed.

All participants performed the rating tasks in two conditions. For the hearing aid users, these two conditions were with (aided) and without their hearing aids (unaided). The normal-hearing participants completed the tasks in a normal listening condition and in a condition with simulated hearing loss (data of the latter condition are not reported here). In each listening condition, participants rated all stimulus utterances, so each participant rated each utterance twice. The order of the arousal/ratings rating tasks and listening conditions were counterbalanced across participants. Two different lists were used to present listeners with a different order of the stimuli 
in the two listening conditions. There was a short break between each of the four blocks (i.e., between the two listening conditions and between the two rating tasks).

\subsection{Acoustic Parameters}

Affect ratings provided by the participants in our study were related to four acoustic parameters which are traditionally related to affective prosody: mean F0 (e.g., Hammerschmidt and Jürgens 2007), mean intensity (e.g., Aubergé and Cathiard 2003), global temporal aspects (Mozziconacci and Hermes 2000), and spectral measures, which are related to vocal effort (e.g., Tamarit et al. 2008). In the current study, mean F0 and mean intensity were calculated for each utterance by averaging over the utterance using Praat (Boersma and Weenink 2013). As a measure of tempo, articulation rate was calculated by dividing the number of syllables in the canonical transcription of the utterance by the file length, excluding pauses longer than $100 \mathrm{~ms}$. Spectral slope is reflected in the spectral information described by the Hammarberg Index (Hammarberg et al. 1980), which is defined as the intensity difference between the maximum intensity in a lower frequency band [0-2000 Hz] versus that in a higher frequency band [2000-5000 Hz]. In this study, the Hammarberg Index energy distribution measure was averaged across the entire utterance.

\section{Results}

The data were analyzed using $\mathrm{R}$ statistical software ( $\mathrm{R}$ Development Core Team 2008). To investigate (a) whether hearing loss severity modulates affect ratings and (b) whether wearing a hearing aid makes listeners more sensitive to subtle differences in acoustic parameters, we compared affect ratings (the dependent variable) of the hearing-impaired listeners in the aided and unaided conditions using linear mixed-effects regression analyses with random intercepts for stimulus and participant. The initial models (one for arousal and one for valence) allowed for three-way interactions between listening condition (aided, unaided), individual hearing loss, and each of the acoustic parameters (mean F0, mean intensity, articulation rate, Hammarberg Index). Interactions and predictors that did not improve model fit (according to the Akaike Information Criterion) were removed using a stepwise exclusion procedure. Interactions were removed before simple effects, and those with the highest non-significant $\mathrm{p}$-values were excluded first.

To investigate whether the use of a hearing aid restores affect perception to the level of normal-hearing older adults, we compared hearing aid users' performance in the aided condition to that of the normal-hearing listeners. The method and model-stripping procedure were identical to that of the first analysis. The initial models (for arousal and valence, respectively) allowed for two-way interactions between group (hearing aid users aided, normal hearing) and each of the four acoustic parameters. 


\subsection{Aided Versus Unaided Listening}

For arousal, mean intensity was found to be a strong cue for arousal rating $\left(\beta=6.606 \times 10^{-2}, \mathrm{SE}=1.528 \times 10^{-2}, p<0.001\right)$ : higher intensity was associated with higher ratings of arousal in the aided and unaided conditions. Moreover, arousal ratings were generally higher in the aided condition than in the unaided condition (mapped on the intercept) $\left(\beta=7.156 \times 10^{-2}, \mathrm{SE}=2.089 \times 10^{-2}, p<0.001\right)$. Significant interactions between listening condition and articulation rate $\left(\beta=3.012 \times 10^{-2}\right.$, $\left.\mathrm{SE}=1.421 \times 10^{-2}, p<0.05\right)$ and listening condition and vocal effort $\left(\beta=1.459 \times 10^{-2}\right.$, $\left.\mathrm{SE}=3.949 \times 10^{-3}, p<0.001\right)$ were observed: while vocal effort and articulation rate did not influence ratings in the unaided condition, their effects were larger in the aided condition. In the unaided condition, those with poorer hearing had lower ratings $\left(\beta=-9.772 \times 10^{-3}, \mathrm{SE}=4.093 \times 10^{-3}, p<0.05\right)$ than those with better hearing, but this was less the case in the aided condition $\left(\beta=7.063 \times 10^{-3}, \mathrm{SE}=2.459 \times 10^{-3}\right.$, $p<0.01)$. This suggests that wearing the hearing aid made the rating patterns of poorer and better-hearing participants more alike. Furthermore, those with poorer hearing associated increases in $\mathrm{F} 0\left(\beta=6.093 \times 10^{-5}, \mathrm{SE}=2.094 \times 10^{-5}, p<0.01\right)$ and in articulation rate $\left(\beta=1.833 \times 10^{-3}, \mathrm{SE}=8.952 \times 10^{-4}, p<0.05\right)$ more with higher arousal than those with better hearing across listening conditions. This suggests that, among the hearing aid users, those with poorer hearing used additional prosodic cues compared to those with relatively good hearing.

For valence, a significant simple effect of mean F0 $\left(\beta=-4.813 \times 10^{-3}\right.$, $\left.\mathrm{SE}=8.856 \times 10^{-4}, p<0.001\right)$ was found: higher pitch was associated with lower ratings, i.e., more negative ratings. None of the other acoustic parameters was predictive of the valence ratings. Moreover, importantly, no effects for listening condition and hearing loss were observed: valence ratings were independent of whether the participants wore their hearing aids or not and were independent of individual hearing loss.

\subsection{Aided Listening Versus Normal-Hearing Controls}

Similar to the previous arousal analysis, a significant simple effect of mean intensity $(\beta=0.071, \mathrm{SE}=0.014, p=0.001)$ was found: higher mean intensity was associated with higher arousal ratings. Although ratings of the hearing aid users did not differ significantly from the normal-hearing participants (mapped onto the intercept, $\beta=-0.030, \mathrm{SE}=0.053, p=0.57$ ), use of mean intensity differed between the two listener groups: hearing aid users responded more strongly to differences in intensity than participants with age-normal hearing $(\beta=0.009, \mathrm{SE}=0.004, p<0.05)$.

For valence, similar to the previous analysis, mean F0 was associated with lower valence ratings $\left(\beta=-4.602 \times 10^{-3}, \mathrm{SE}=1.168 \times 10^{-3}, p<0.01\right)$. No other acoustic parameters were predictive of the valence ratings. There was no effect of group, nor any interactions between group and the acoustic parameters. 


\section{Discussion}

This study aimed to investigate whether the use of a hearing aid restores affect perception to the level of older adults with age-normal hearing. More specifically, our study investigated to what extent hearing aids and individual hearing loss modify sensitivity to the acoustic parameters cueing affect in older hearing aid users.

The study showed that the hearing aid restored affect perception in the sense that the use of the hearing aid makes rating patterns of hearing aid users with severe hearing loss more similar to those with less severe hearing loss. Secondly, the study showed that the use of a hearing aid changed the pattern of acoustic parameters that were used for arousal perception. Importantly, across the aided and unaided conditions, hearing loss modulated the extent to which listeners used alternative cues to interpret arousal (i.e., other cues than intensity): hearing-impaired listeners with more severe degrees of hearing loss made more use of articulation rate and mean F0. In other words, gradually acquired hearing loss causes listeners to rely on different cues for their interpretation of arousal, but restoring their hearing by means of a hearing aid will also change which cues they rely on for their interpretation of arousal. Older adults may only start using additional cues (such as articulation rate) for their interpretation of arousal with more severe hearing loss. In a related study (Schmidt et al., submitted), older adults with mild hearing loss were tested who were not wearing hearing aids. For this group with mild hearing impairment, intensity emerged as the only significant predictor of arousal. Note, however, that this reliance on multiple cues rather than on a single cue does not hold for valence, where F0 is the only prosodic cue listeners irrespective of their hearing sensitivity are using.

Hearing aid users wearing their hearing aid generally showed the same pattern of affect ratings as participants with age-normal hearing, especially for the valence dimension. However, for arousal ratings, those wearing a hearing aid were actually more sensitive to intensity differences than participants in the reference group. This may be because hearing in the reference group was normal for their age, but still implied elevated high-frequency thresholds. Consequently, older adults in the reference group were less sensitive, at least to some acoustic differences, than the hearing aid users.

In sum, the current study shows that older hearing aid users do not generally differ from their normal-hearing peers in their perception of arousal and valence, which underlines the importance of hearing aids in the rehabilitation of affect perception. While the perception of valence seems to be independent of listening condition and individual hearing loss, wearing hearing aids matters for the interpretation of rating prosodic information related to arousal. Due to this difference between emotion dimensions, future studies on affect perception in hearing aid users should treat perception of arousal and valence separately.

Acknowledgments This project has received funding from the European Union's Seventh Framework Programme for research, technological development and demonstration under grant agreement no FP7-PEOPLE-2011-290000. The Netherlands Organization for Scientific Research 
(NWO) supported the research by Esther Janse (grant number 276-75-009) and Odette Scharenborg (grant number 276-89-003).

Open Access This chapter is distributed under the terms of the Creative Commons AttributionNoncommercial 2.5 License (http://creativecommons.org/licenses/by-nc/2.5/) which permits any noncommercial use, distribution, and reproduction in any medium, provided the original author(s) and source are credited.

The images or other third party material in this chapter are included in the work's Creative Commons license, unless indicated otherwise in the credit line; if such material is not included in the work's Creative Commons license and the respective action is not permitted by statutory regulation, users will need to obtain permission from the license holder to duplicate, adapt or reproduce the material.

\section{References}

Aubergé V, Cathiard M (2003) Can we hear the prosody of smile? Speech Comm 40:87-97

Banse R, Scherer KR (1996) Acoustic profiles in vocal emotion expression. J Pers Soc Psychol 70(3):614-636

Boersma P, Weenink D (2013). Praat: doing phonetics by computer. www.praat.org/. Accessed 10 April 2013

Bradley MM, Lang PJ (1994) Measuring emotion: the self-assessment manikin and the semantic differential. J Behav Ther Exp Psychiatry 25(1):49-59

Coutinho E, Dibben N (2013) Psychoacoustic cues to emotion in speech prosody and music. Cogn Emot 27(4):658-684

Dupuis K, Pichora-Fuller MK, Chasteen AL, Marchuk V, Singh G, Smith SL (2015) Effects of hearing and vision impairments on the Montreal cognitive assessment. Aging Neuropsychol Cognit: J Norm Dysfunct Dev 22(4):413-437

Grant KW (1987) Identification of intonation contours by normally hearing and profoundly hearing-impaired listeners performance. J Acoust Soc Am 82(4):1172-1178

Grimm M, Kroschel K, Narayanan S (2008). The Vera am Mittag German audio-visual emotional speech database. In Proceedings of the IEEE International Conference on Multimedia and Expo (ICME) (pp 865-868). Hannover, Germany

Hammarberg B, Fritzell B, Gauffin J, Sundberg J, Wedin L (1980) Perceptual and acoustic correlates of abnormal voice qualities. Acta Otolaryngol 90:441-451

Hammerschmidt K, Jürgens U (2007) Acoustical correlates of affective prosody. J Voice: Off J Voice Found 21(5):531-540

International Organization for Standardization (2000) Acoustics - statistical distribution of hearing thresholds as a function of age. ISO 7029. ISO, Geneva

Most T, Michaelis H (2012) Auditory, visual, and auditory-visual perceptions of emotions by young children with hearing loss versus children with normal hearing. J Speech Lang Hear Res 55(4):1148-1162

Most T, Weisel A, Zaychik A (1993) Auditory, visual and auditory-visual identification of emotions by hearing and hearing-impaired adolescents. Br J Audiol 27:247-253

Mozziconacci SJL, Hermes DJ (2000) Expression of emotion and attitude through temporal speech variations. In Sixth International Conference on Spoken Language Processing (pp 373-378). Beijing, China

Nasreddine ZS, Phillips NA, Bedirian V, Charbonneau S, Whitehead V, Collin I, Cummings JL, Chertkow H (2005) http://www.mocatest.org/. Accessed 28 Nov 2013

Orbelo DM, Grim MA, Talbott RE, Ross ED (2005) Impaired comprehension of affective prosody in elderly subjects is not predicted by age-related hearing loss or age-related cognitive decline. J Geriatr Psychiatry Neurol 18(1):25-32 
Paulmann S, Pell MD, Kotz SA (2008) How aging affects the recognition of emotional speech. Brain Lang 104(3):262-269

R Development Core Team (2008) R: a language and environment for statistical computing. R Foundation for Statistical Computing, Vienna. http://www.R-project.org/. Accessed 4 March 2013

Rigo TG, Lieberman DA (1989) Nonverbal sensitivity of normal-hearing and hearing-impaired older adults. Ear Hear 10(3):184-189

Scherer KR (1986) Vocal affect expression: a review and a model for future research. Psychol Bull 99(2):143-165

Scherer KR (2003) Vocal communication of emotion: a review of research paradigms. Speech Comm 40(1-2):227-256

Scherer KR, Banse R, Wallbott HG (2001) Emotion inferences from vocal expression correlate across languages and cultures. J Cross Cult Psychol 32(1):76-92

Tamarit L, Goudbeek M, Scherer K (2008). Spectral slope measurements in emotionally expressive speech. In Proceedings for ISCA ITRW Speech Analysis and Processing for Knowledge Discovery. Aalborg, Denmark

Waldron-Perrine B, Axelrod BN (2012) Determining an appropriate cutting score for indication of impairment on the Montreal Cognitive Assessment. Int J Geriatr Psychiatry 27(11):1189-1194

Wilting J, Krahmer E, Swerts M (2006) Real vs. acted emotional speech. In Proceedings of the 9th International Conference on Spoken Language Processing (Interspeech) (pp 805-808). Pittsburgh, Pennsylvania 